\title{
Ökonomische und außerökonomische Werteorientierung in der Organisationswahl*
}

\author{
CHRISTOPH SCHANK $^{* *}$
}

Vor dem Hintergrund des Eintrittes in das duale System der Berufsausbildung werden die Wahlentscheidungen für oder gegen bestimmte ausbildende Organisationen unter dem Gesichtspunkt verschiedener, alternierender oder miteinander in Konkurrenz stehender Werteorientierungen untersucht. Es wird dadurch mit der Vorstellung gebrochen, die ökonomische Handlungslogik sei der einzig legitime Ausdruck rationalen Handelns. Unter Zuhilfenahme einer empirischen Vorgehensweise werden jene Werteorientierungen beschrieben und ihr Einfluss auf die Organisationswahl bestimmt. Es zeigt sich dabei, dass sich nicht allein vermeintlich systemkonforme, ökonomische Wahlmotive als handlungsleitend - und damit für ein belastbares Erklärungsmodell als hinreichend - erweisen.

Schlagwörter: Werteorientierung, Rational Choice, Berufsausbildung, Mikrosoziologie

\section{Economic and Non-Economic Value Orientation within the Selection of Organizations}

Against the background of entering the dual system of vocational training, the decisions for and against certain educational organizations will be investigated in consideration of different alternate or competing value orientations. Thus, the idea of economic behavioral logic being the only legitimate expression of rational choice is abandoned. By utilizing an empirical approach, these value orientations and their effect on the selection of specific organizations will be determined. It shows that the choices are not exclusively informed by allegedly system-compatible, economic motives; therefore, these motives cannot be deemed sufficient to support an explanatory model.

Keywords: Value Orientations, Rational Choice, Vocational Training, Microsociology

\section{Forschungsleitung und Hinführung}

Der demografische und gesellschaftliche Wandel in den entwickelten Industrienationen und seine inzwischen breit und eindringlich diskutierten Folgen für die sozialen Sicherungssysteme, das Bildungssystem und den Arbeitsmarkt haben das Bewusstsein für die Knappheit qualifizierter Fachkräfte geschärft. Im Werben um das begehrte Humankapital stellen Unternehmen fest, dass diese im Arbeitsprozess verwertbaren Fertigkeiten und Kenntnisse untrennbar mit einer natürlichen Person verbunden sind

Beitrag eingereicht am 14.06.2011; nach doppelt verdecktem Gutachterverfahren überarbeitete Fassung angenommen am 10.4.2012.

** Dr. Christoph Schank, Institut für Wirtschaftsethik, Universität St. Gallen, Tannenstrasse 19, CH-9000 St. Gallen, Tel.: +41 71224 3147, Fax: +41 71224 2881, E-Mail: christoph.schank @unisg.ch, Forschungsschwerpunkte: Corporate Social Responsibility, Corporate Citizenship, Bildungs- und Arbeitsmärkte. 
und nicht losgelöst von ihr gehandelt werden können. Dadurch wird die Frage aufgeworfen, welche Werte und Präferenzen sich bei der Wahl eines Arbeitgebers tatsächlich als entscheidend erweisen. Das neoklassische Basismodell zieht sich dabei überwiegend auch nach allen humankapitaltheoretischen Ergänzungen auf einen Standpunkt zurück, nach dem monetär bewertbare Erträge und Kosten die Hauptlast der Erklärung tragen, während nicht-monetäre Überlegungen bagatellisiert werden (vgl. Hinz/Abraham 2005: 32ff.).

In diesem Beitrag soll daher mit Hilfe eines empirischen Designs der Frage nachgegangen werden, ob die Organisationswahl einen allein von ökonomischen Motiven geleiteten Prozess darstellt oder ob hier außerökonomische Werte maßgeblich zum Tragen kommen. Der dabei gewählte Untersuchungskontext ist jener des dualen Systems der Berufsausbildung, das den Übergang von der (rein) schulischen in die berufliche Ausbildung und das Erwerbsleben markiert. Aus einer unternehmensethischen Perspektive ist dieser Schwellenübergang deshalb von besonderer Bedeutung, da die (potenziellen) Unternehmensmitglieder an einer Risikopassage stehen und Unternehmen folglich im Umgang mit jugendlichen Berufseinsteigern, die für ihre berufsbiografische Entwicklung die ersten Weichen stellen, eine besondere Verantwortung tragen. Ein Verständnis der wahlentscheidenden Werte und Präferenzen ist daher nicht nur für die zielgerichtete Ansprache zukünftiger Fachkräfte, sondern auch für die Herstellung einer bestmöglichen Passung von organisationalen und individuellen Wertehaltungen von Bedeutung.

Dieser Beitrag untersucht die Entscheidung für oder gegen eine bestimmte Organisation als betrieblichen Lernort. In einem eher weiten Sinne ist darin ein Diskussionsbeitrag in der Tradition der deskriptiven Ethik zu verstehen, da für eine abgrenzbare Gruppe handlungsleitende Werteorientierungen herausgestellt werden, denen durchaus ein sittlicher Gehalt attestiert werden kann, ohne dass über diesen Gehalt in diesem Beitrag aus einer normativen Perspektive kommend geurteilt werden soll (vgl. Göbel 2010: 14f.). Unter der Werteorientierung soll an dieser Stelle eine persönliche oder gruppenbezogene Auffassung des Erstrebenswerten und der daraus folgenden Konsequenzen für Einstellung und Handlung verstanden werden. ${ }^{1}$ Werteorientierungen sind damit zwingend auch in wissenschaftlichen Disziplinen wie der Ökonomie vorhanden, die für sich selbst dadurch eine Wertfreiheit beanspruchen, indem Marktgesetze wie alternativlose, überpositive Naturgesetze formuliert werden. Gleichsam wird davon ausgegangen, dass der Wertehimmel der Akteure kein homogener, sondern ein pluralistischer, konkurrierender aber gleichsam kontextualisierter ist.

Mit Hilfe einer methodentriangulierten empirischen Vorgehensweise soll die Frage erörtert werden, an welchen Werten sich (zumeist jugendliche) Berufs- und Organisationswählende an der Schwelle des Überganges in die Arbeitswelt orientierten und ob hier die alleinige Deutungshoheit den (bildungs-)ökonomischen Theorieansätzen und der daraus abzuleitenden Werteorientierung zukommen kann. Diese ökonomische (Werte-)Orientierung, die sich aus den Marktgesetzen ableitet und folglich dem rational handelnden Akteur oktroyiert ist, soll dabei als systemkonform verstanden werden.

$1 \quad$ Soweit erfolgt die Definition in Anlehnung an Kluckhohn (1951). 
Stärker ausdifferenziert liegt das Erkenntnisinteresse dieses Beitrages in folgenden Fragen begründet:

(1) Welche Werteorientierung erscheint angesichts der Organisationswahl im Kontext des dualen Systems der Berufsausbildung aus (bildungs-)ökonomischer Sicht als rational respektive systemkonform?

(2) Gibt es dazu konkurrierende Werteorientierungen, denen aus der Perspektive der ökonomischen Marktlogik heraus der Charakter der Rationalität abgesprochen werden müsste?

(3) Um welche Werteorientierungen handelt es sich dabei und wie können diese gegebenenfalls gegeneinander abgegrenzt werden?

(4) Inwiefern beeinflussen Werteorientierungen als Ausdruck der Einstellung gegenüber Organisationstypen tatsächlich das overte, d.h. unverdecktbeobachtbare Verhalten?

Im anschließenden zweiten Kapitel wird die Frage aufgeworfen, inwiefern bei der Entscheidung für oder gegen eine ausbildende Organisation von einer rational begründeten Wahl gesprochen werden kann. Nach dieser knappen Vorklärung werden Forschungslinien skizziert, die für den weiteren Verlauf ins Kalkül gezogen werden. In zwei Unterkapiteln werden ökonomische und außerökonomische Werteorientierungen diskutiert. Kapitel 3 ist der Beschreibung des empirischen Vorgehens und den dadurch aufgeworfenen Resultaten gewidmet. Über eine explorative Faktorenanalyse wird dabei geprüft, ob sich bestimmte Werte zu einer Wertehaltung summieren lassen. Im Rahmen einer Modellierung in der Tradition einer weiten Rational-Choice-Theorie wird danach regressionsanalytisch überprüft, ob sich individuelle Wertehaltungen tatsächlich als entscheidungsleitend erweisen. Der Beitrag schließt mit einer Diskussion in Kapitel 4.

\section{Der Weg in die Organisation: rationale Entscheidung oder gesellschaft- liche Zuweisung?}

Die Frage, ob eine bestimmte ökonomische oder außerökonomische Werteorientierung für die tatsächlichen Allokationsfolgen, d.h. das Matching von Auszubildenden und Ausbildungsbetrieb, überhaupt relevant sein kann, birgt die Problematik eines methodologischen Grundverständnisses vom Menschen, das - sofern nicht thematisiert - unkontrolliert im Hintergrund wirkt. Gerade im Kontext der Berufseinmündung konkurrieren verschiedene Grundannahmen über die Autonomie des Akteurs, zu deren Systematisierung auch von den „ökonomischen“ und den „,soziologischen“ Traditionen gesprochen wird ${ }^{2}$. Während die sogenannte ökonomische Tradition eng mit dem Wirken Beckers und seiner ökonomischen Erklärung menschlichen Verhaltens (vgl. Becker 1993) verbunden ist, werden unter der soziologischen Perspektive hingegen Ansätze subsummiert, welche das ökonomische Menschenbild des Homo

2 Die Bezeichnung der „soziologischen Ansätze“ ist hier insofern überaus unglücklich, da gerade in jüngster Zeit Rational-Choice-Ansätze in der Soziologie an Gewicht gewinnen und sich durch eine Nähe zur ökonomischen Tradition auszeichnen. Grundsätzlich ist es daher treffender, statt von soziologischen von allokationstheoretischen Ansätzen zu sprechen. 
oeconomicus durch jenes des Homo sociologicus ersetzen. Als Vertreter dieser Tradition charakterisiert Scharmann (1965: 14) die Vorgänge der Berufseinmündung als
„in hohem Maße abhängig von den allgemeinen kulturellen und sozialen Bedin- gungen, von der jeweiligen Wirtschaftslage und von den familiären Verhältnis- sen des Berufsanwärters, also von allgemeinen Bedingungen und Faktoren, auf die der einzelne meist nur einen geringen Einfluss hat."

Mit dem Verweis auf eine Organisationswahl - und nicht etwa einer Zuweisung (vgl. Beck et al. 1979) oder Findung (vgl. Lappe 2006: 75) - trifft der vorliegende Beitrag daher bereits eine Aussage über anthropologische und normative Grundannahmen gleichermaßen: Der Akteur wird als rational entscheidendes Wesen angenommen, das Informationen und Ressourcen planmäßig erwirkt und zum Einsatz bringt, um schlussendlich seinen persönlichen Nutzen so weit wie möglich zu maximieren.

In diesem Beitrag soll daher nicht die Sinnlogik des Marktes und der Ökonomik selbst hinterfragt werden, wohl aber daraus abgeleitete Prämissen über die Werteorientierung. Anders gewendet steht die Frage im Vordergrund, worin sich persönlicher Nutzen benennen lässt und wie dieser im Spiegel unterschiedlicher Werteorientierungen Niederschlag findet.

In diesem Beitrag werden vier Forschungslinien in Betracht gezogen: bildungsökonomische Ansätze, die Personalimageforschung, Ansätze der Berufswahlforschung und das Ausbildungsmarketing. Die (bildungs-)ökonomischen Ansätze zur Erklärung einer Organisationswahl im Bildungs- und Ausbildungskontext stellen den ersten Anlaufpunkt zur Erklärung von Werteorientierungen dar. Flankierend dazu können diese Ansätze im Vergleich mit Beiträgen zur Personalimageforschung reflektiert werden, die das Hauptaugenmerk auf Attraktivitätsunterschiede von arbeitgebenden Organisationen legen und hierfür eine breite empirische Datengrundlage bieten (vgl. etwa Lieber 1995; Sutherland et al. 2002; Simon et al. 1995; Berthon et al. 2005). Die Eignung dieser Ansätze für die hier diskutierte Thematik wird jedoch dadurch maßgeblich erschwert, dass die Personalimageforschung überwiegend auf Hochschulabsolventen oder Führungskräfte fokussiert ist und dabei vernachlässigt, dass der Einstieg in das duale System eine eigene Bildungs- und Lernsituation darstellt. Gerade sehr stark empirisch und praxisnah ausgerichtete Studien aus angelsächsischen Ländern, die über kein etabliertes System einer solchen Berufsausbildung verfügen, sind daher nur bedingt geeignet. Ähnlich beschränkt bleiben die Zugänge, die gerade im deutschsprachigen Raum sehr umfassende Berufswahlforschung bietet (vgl. exemplarisch Allehoff 1985; Beinke 2006; Lange 1978). Zwar ist gerade die Kontexteinbettung sehr ergiebig, jedoch hat dieses einst sehr vitale Forschungsfeld mit der Entwicklung der Arbeitswelt nicht schrittgehalten und den Höhepunkt seiner Schöpfungskraft überschritten (vgl. Nowak 2002). Entsprechend wenig aktuell sind Theorien und Befunde. Ein zweites Problem ist ein inhaltliches, nämlich die fast ausschließliche Konzentration auf die Wahl des Berufs(bildes) bei einer gleichzeitigen Vernachlässigung des Ausbildungsunternehmens. Hier existieren nur vereinzelte Befunde, die in die vorliegende Diskussion einfließen können. Schlussendlich kann mit dem Ausbildungsmarketing eine mögliche Scharnierfunktion zwischen der Personalimageforschung und der Berufswahlforschung ausgemacht werden. Obwohl hier unter dem Eindruck des demografischen 
Wandels eine zunehmende Dynamik festzustellen ist, bleibt das Literaturfeld aber noch übersichtlich (vgl. Ebbinghaus 2011; Gertz 2011; Sager 2008).

Für den weiteren Verlauf bilden die (bildungs-)ökonomischen Ansätze die theoretische Grundlage, während Beiträge der übrigen Forschungslinien punktuell herangezogen werden.

\subsection{Beitrag und Grenzen ökonomischer Erklärungen der Organisationswahl}

Zur Betrachtung der Partizipationsmotive und Wahlentscheidungen am dualen System der Berufsausbildung erscheint das Heranziehen ökonomischer Theorien zuerst einmal nur folgerichtig. Die auf diesem Wege erworbenen Berufsabschlüsse sind eine am Markt gehandelte Ware (vgl. Beck et al. 1980: 20) und als solche der Marktlogik des Tausches von Einkommen gegen Verfügungsrechte an einem Arbeitsvermögen unterworfen. Berufsabschlüsse sind auf einem noch immer berufsständisch geordneten Arbeitsmarkt eine „harte Währung“ (Frommberger 2007: 149), die sich (im Idealfall) über die gesamte Spanne der Erwerbstätigkeit hinweg in ökonomisches Kapital konvertieren lässt. Es liegt demnach zu Beginn der Überlegungen nahe, die Entscheidung für oder gegen eine bestimmte Organisation - oder eine abgrenzbare, merkmalsähnliche Gruppe an Organisationen - unter dem Gesichtspunkt einer Investition in das eigene Humankapital zu erörtern. Die Wahl der ausbildenden Organisation ist nun aus humankapitaltheoretischer Sicht allein daher bedeutsam, da das in der Ausbildung erworbene Humankapital nur bedingt über die Organisationsgrenzen hinaus transportierbar ist. Dies gilt umso deutlicher, wenn dabei staatliche und kulturelle Grenzen überschritten werden (vgl. Friedberg 2000). Becker (1993: 33ff.) unterscheidet hierbei zwischen Investitionen in das allgemeine und das spezifische Humankapital. Während allgemeines Humankapital, resultierend aus dem general training, im Idealfall am Markt ohne Einbußen transferiert werden kann, ist spezielles Humankapital, wie es aus dem specific training hervorgeht, in vollem Umfang lediglich in jener Organisation einsetzbar, in der es erworben wurde.

Die unterstellte Werteorientierung der ökonomischen Ansätze ist eine, die dem (Lebens-)Einkommen die zentrale, wenn nicht alleinige Geltung zur Erklärung des Verhaltens zukommen lässt. Becker selbst merkt dabei sogar an, dass auch nicht unmittelbar monetär relevante Motive und Werte die Organisationswahl beeinflussen oder sogar entscheiden können (vgl. Becker 1993: 21). Durchaus diplomatisch hält er an anderer Stelle fest: „,Some working time would be supplied to the lower paying occupation if the difference in earnings were offset by a difference in utility"(Becker 2008: 166). Gleichwohl liefert er darüber hinaus gehend kaum Ansätze, die weitere Werteorientierungen fundieren könnten. Schmalen (1973: 295), dessen Interesse der Erklärung der Berufswahl in Deutschland gilt, stellt der monetären Dimension lediglich „sonstige Nettovorteilsdominanten“ zur Seite und marginalisiert damit andere Werteorientierungen spürbar. Noch weiter fortgeschritten findet sich diese Sicht bei Sheldon (1986), der in außerökonomischen Motiven nicht zu operationalisierende Störgrößen - und damit einen eher methodisch lästigen Ausdruck der bedingten Akteursrationalität - zu erkennen glaubt. Selbst die mikrofundierte Soziologie von Esser (2000: 71), die unterschiedliche empirisch oder theoretisch abgeleitete Präferen- 
zen zulässt, versteift sich dabei in der Annahme, die entscheidungsleitenden Werte der Akteure seien grundsätzlich auf Geld und Ansehen kondensierbar.

Als Zwischenresultat der Betrachtung (bildungs-)ökonomischer Ansätze zur Erklärung rationalen Handelns kann exponiert werden, dass besagtes Handeln stets unter der Prämisse der Marktfähigkeit und Marktpositionierung beschrieben und damit auf eine solche Werteorientierung verengt wird, die sich mittel- oder unmittelbar, in Gegenwart oder Zukunft monetär niederschlägt. Zwar werden flankierende Werteorientierungen keineswegs negiert, sind aber meiner Meinung nach häufig lediglich als methodologisches Feigenblatt aufzufassen, mit dem im Federstreich der conditio humana, d.h. dem Menschen als soziales, in reziproke und nicht-marktliche Beziehungen eingebettetes Kulturwesen Rechnung getragen werden soll.

Aus den humankapitaltheoretischen Ansätzen lässt sich eine Reihe von einzelnen Valenzen folgern, die zusammengenommen eine ökonomische Werteorientierung bilden und mit deren Verfolgung sich der Akteur am Ausbildungs- und Arbeitsmarkt sozusagen systemkonform verhalten würde. Im Kern zielen diese Teilaspekte darauf ab, das Nettoeinkommen über die gesamte Lebenserwerbsphase hinweg zu maximieren. Es handelt sich hierbei um

(1) Ausbildungsvergütung: Das während der Ausbildungsphase gezahlte Entgelt schlägt sich unmittelbar in der Berechnung des Nettoeinkommens nieder.

(2) Arbeitsmarktmobilität: Die Wahrscheinlichkeit, nach der Ausbildung an andere Organisationen anschlussfähig zu bleiben. Eine grundsätzliche Mobilität ist allein schon deshalb gegeben, da das duale System der Berufsausbildung zu hoch formalisierten, anerkannten Abschlüssen führt, die nicht an die ausbildende Organisation gebunden sind. Dennoch ist anzunehmen, dass bestimmte Organisationen aufgrund ihrer Struktur und ihres Geschäftsfeldes eher betriebsspezifisches, weniger gut transferierbares Humankapital generieren, wohingegen andere Organisationen solches bereitstellen, das stärker in der Breite Verwendung finden kann.

(3) Übernahmemöglichkeiten: Der Übergang von der Ausbildung in die (reguläre) Erwerbstätigkeit kann in der ausbildenden Organisation erfolgen. Eine möglichst wahrscheinliche Übernahme reduziert die Gefahr von Erwerbsausfällen im Anschluss an die Ausbildung.

(4) Weiterbildungsmöglichkeiten: Durch die Organisation beförderte Weiterbildungen nach der Berufsausbildung stellen Investitionen in das Humankapital dar, die zu einem Produktivitätszuwachs führen und sich somit auf das Einkommen auswirken.

(5) Karriere- und Entwicklungspotential: Die Möglichkeit zum innerorganisationalen Aufstieg offeriert spürbare Einkommensanstiege gegenüber solchen Organisationen, die aufgrund ihrer Unternehmensgröße oder -struktur keine hierarchische Entwicklung zulassen.

(6) Vereinbarkeit von Familie und Beruf: Dieses Merkmal nimmt eine Vermittlerrolle zwischen ökonomischen und außerökonomischen Werten ein, da eine Erfüllung in der Familie nur unzureichend über die ökonomischen Theorien abzudecken ist. Dennoch wird es hier zu den ökonomischen Werten gezählt, da eine möglichst umfangreich gegebene Vereinbarkeit von Familie und Beruf Verdienstausfälle in 
Form eines (stark) reduzierten Arbeitsvolumens oder einer mehrjährig unterbrochenen Berufstätigkeit unwahrscheinlicher werden lässt. In den begleitenden problemzentrierten Interviews (vgl. Kapitel 3.1) wurde bestätigt, dass mit einer hohen Vereinbarkeit von Beruf und Familie primär die Möglichkeit zu einer Berufsrückkehr oder einer zur Erziehungstätigkeit begleitenden Erwerbstätigkeit verstanden wird und damit die ökonomische Perspektive überwiegt.

\section{2 Über die Grenzen der ökonomischen Theorie hinaus}

Gegen diese einseitig zugrunde gelegte Werteorientierung verwehren sich nicht allein induktiv-empirisch vorgehende Disziplinen, sondern auch, wenngleich weniger kontextuell explizit, die Wirtschaftsethik. Wenn Ulrich (2005: 10ff.) davor warnt, das gesamte Leben unter die Sachlogik des Marktes zu stellen und damit jegliches menschliche Zusammenleben als eine „Marktgesellschaft“ verstehen zu wollen, deutet er damit zugleich die Grenzen einer rein ökonomischen Betrachtung der Vorgänge der Organisationswahl an. ${ }^{3}$ Zwar finden der Erwerb und die Verwertung von Humankapital durchaus auf Märkten statt, jedoch stellt, wie oben bereits genannt, gerade die Entscheidung für einen Ausbildungsberuf und ein Ausbildungsunternehmen einen nicht nur berufsbiografisch höchst relevanten Moment dar. Mehr noch ist die exponiert deutliche Interpenetration des Bildungs- und Arbeitsmarktes mit anderen gesellschaftlichen Subsystemen (vgl. Göbel 2010: 87) ein Alleinstellungsmerkmal, welches das Tauschgut „Verfügungsrecht über Arbeit“ von anderen Faktor- und Gütermärkten separiert. Eine Analyse der Werteorientierung im Kontext der Organisationswahl kann daher nicht ohne Berücksichtigung solcher Werte und Präferenzen geschehen, die nicht auf Märkten getauscht oder unmittelbar monetär verwertbar gemacht werden können. ${ }^{4}$

Stärker lebensweltlich eingebettete Betrachtungen der Organisationswahl im Kontext der Berufsausbildung sind insofern selten und spezifisch, da sie sowohl das kulturelle und rechtliche Gefüge der deutschen Berufsbildungsinstitutionen berücksichtigen als auch von einem rationalen Akteur ausgehen müssen, um an die hier angestellten Überlegungen anschlussfähig zu bleiben.

Einen Schritt hin zu einem „multiple attribute model of choice“ beschreitet Austen (1997) am Beispiel der Berufseinmündung in Australien. Zwar lassen sich angesichts unterschiedlicher Rahmenbedingungen der Arbeitsmärkte hieraus keine unmittelbaren Valenzen ableiten, die Autorin verdeutlicht aber die Möglichkeit, monetäre, nichtmonetäre und Kontextvariablen in einem Modell bedingter Rationalität zu integrieren.

$3 \quad$ Ulrich kritisiert primär die vermeintliche Wertfreiheit der ökonomischen Handlungslogik, nicht die mit ihr verfolgten (Ober-)Ziele. Diese ergeben sich aber nach der ökonomischen Betrachtung zwingend aus den Tauschgütern des (Arbeits-)Marktes (Arbeitsvermögen wird gegen Einkommen getauscht).

$4 \quad$ In seinem klassischen Aufsatz zu den verschiedenen Kapitalarten beschreibt Bourdieu (1983) verschiedene Möglichkeiten der Transformation von Kapitalarten untereinander. Dies gleichermaßen ins Kalkül ziehend würde bedeuten, dass auch mit vermeintlich außerökonomischen Werteorientierungen in bisweilen komplizierten Austauschbeziehungen ökonomische Werte realisiert werden können. Jedoch sind solche Transformationsprozesse wiederum mit Kosten verbunden, so dass im Weiteren davon ausgegangen wird, dass solche Transformationen von den Akteuren nicht intendiert werden. 
Vergleichbar geht auch Neubäumer (1999) vor, wenn sie die monetären Dimensionen humankapitaltheoretischer Ansätze in „Variablen der Wettbewerbsfähigkeit“ integriert. Auch unter Einbezug stärker induktiv-qualitativ ausgerichteter Theorien rechnet sie zu diesen Variablen (a) das erzielbare Einkommen, (b) Weiterqualifizierungsund Aufstiegsmöglichkeiten, (c) Art und Inhalt der Tätigkeit, (d) Arbeitsdruck, (e) physische Belastung am Arbeitsplatz und (f) Wechsel- oder Nachtschichten. Die starke Betonung des Arbeitsleides verdeutlicht hier den möglichen Kompensationseffekt der Entlohnung für inhaltliche Defizite, nicht nur - wie von der klassischen Ökonomie angenommen - für das aufgebrachte Arbeitsvolumen. Obwohl anhand dieser beiden beispielhaften Ansätze die Existenz stärker differenzierter Werteorientierungen im Kontext der Berufsausbildung aufgezeigt werden können, lassen die Arbeiten beider Autorinnen noch keinen Rückschluss auf eventuell konkurrierende Werteorientierungen zu.

Fruchtbar erscheint zudem ein psychologischer Ansatz nach Rosenstiel und Nerdinger (2000), die in ihrer Münchner Wertestudie zwischen der Karriereorientierung, der Freizeitorientierung und dem alternativen Engagement unterscheiden. ${ }^{5}$ Gerade die aufgeführte und empirisch überprüfte Freizeitorientierung verdeutlicht das Vorhandensein einer Werteorientierung, die mit dem ökonomischen Nutzenkalkül der Einkommensmaximierung bricht und lebensweltliche, marktfremde Werte darüber erhebt.

Ein ähnliches Organisationsmuster hinsichtlich der Werteorientierung auf außerorganisationaler, außerberuflicher und damit auch ,außermarktlicher“" Erfüllung zeigt Schein (1980) mit seiner Menschenbildtypologie auf. Dem rational-economic man, der sich durch externe Anreize in seinem Tun motivieren lässt und sich am Markt und in der Organisationswahl systemkonform verhält, stellt er den social man gegenüber, der sich von Beruf und Markt entfremdet hat, das ökonomische Nutzenkalkül als sinnentleert verneint und seine Motivationsstruktur an menschlicher Nähe, Status und Beziehungen ausrichtet, die sowohl inner- als auch außerhalb der Organisation gepflegt werden. Ein derart beschaffener social man erkennt in der Organisationswahl bestenfalls eine Strategie zur Vermeidung von (Arbeits-)Leid, mithin auch eine Wahl, die ihm gesellschaftlich aufgezwungen oder abgenötigt wird.

Aus diesen theoretischen Befunden gestaltet sich die Ableitung von Einzelwerten, die im Zusammenspiel eine konsistente Werteorientierung bedingen, noch immer unbefriedigend. Kapitel 3 vorweggreifend wurde daher auf ein qualitatives Vorgehen zur Exploration möglicher Werte zurückgegriffen. Aus diesen Erhebungen und der begleitenden Rezeption theoretischer Ansätze wurde die in Kapitel 2.1 begonnene Liste der Valenzen wie folgt fortgesetzt:

(1) Ausbildung mit Gleichaltrigen: Die Zusammensetzung der Beschäftigtenstruktur, die für die (jugendlichen) Auszubildenden im unterschiedlichen Umfang den Austausch mit Gleichaltrigen (oder überhaupt anderen Auszubildenden) bietet.

$5 \quad$ Vertiefend dazu die älteren Arbeiten von Rosenstiel (1982), Rosenstiel und Stengel (1987) sowie die vergleichbaren Arbeiten von Dubin (1956) und Dubin und Goldmann (1972). 
(2) Arbeitsbelastung: Das erwartete Arbeitsleid in Form von ungünstigen oder langen Arbeitszeiten.

(3) Internationalität der Ausbildung: Die Möglichkeit, Ausbildungsinhalte grenzübergreifend zu durchlaufen oder besondere Zusatzqualifikationen durch den Aufbau fremdsprachlicher oder interkultureller Kompetenz zu erwerben. Hier ist eine ökonomische Nutzenorientierung denkbar, wenn mit diesen international erworbenen Erfahrungen ein höherwertigerer Karrierepfad verbunden wird. Diese Sichtweise wurde in den qualitativen Interviews (vgl. Kapitel 3.1) zumindest für die Ausbildungssituation aber nicht artikuliert. Da der Schwerpunkt hier klar auf der individuellen, ganzheitlichen Entwicklung liegt, erscheint eine Zuordnung zu den nicht unmittelbar monetär bewertbaren Werten sinnvoll.

(4) Vielseitigkeit der Ausbildung: Der Facettenreichtum der während der Ausbildung durchlaufenen Tätigkeiten, Abteilungen und Geschäftsfelder.

(5) Selbstverwirklichungspotential: Die Möglichkeit zur Entfaltung der eigenen Persönlichkeit im organisationalen Umfeld. Dieses Bedürfnis zur Suche und Entfaltung der eigenen Talente, Fähigkeiten und Neigungen auch und gerade in einem beruflichen Kontext stellt eine klassische Motivationsdimension nach Maslow (1943) dar.

(6) Arbeits- und Betriebsklima: Die Beziehung zu Vorgesetzen und Kollegen, Unternehmens- und Führungskultur.

(7) Persönlichkeit der Betreuung: Das Vorhandensein einer ganzheitlichen Orientierung am Auszubildenden und einer Betreuungsrelation.

Ein weiterer Wert in puncto Reputation und Image, nämlich das Ansehen der Organisation in der relevanten Bezugsgruppe des Freundes- und Familienkreises findet im Rahmen der später vorgestellten Modellierung gesonderte Verwendung (vgl. Kapitel 3.2). Hier wird der von Bourdieu (1998) begründeten Theorie des sozialen Raums gefolgt, nach der die Zugehörigkeit zu einer bestimmten Organisation der Person Status zuweist und als Ausdruck der eigenen Kapitalstruktur gedeutet werden kann. Daraus folgt die Annahme, dass eine solche Organisation gewählt wird, die durch ihr Renommee und ihre Reputation auch das individuelle Ansehen der Organisationsmitglieder stärkt.

Als zugehörige Hypothese wurde aufgestellt, dass die Valenzen 1 bis 5 in ihrem $\mathrm{Zu}$ sammenspiel als ökonomische Werteorientierung von den Organisationswählenden erkannt und von manchen auch primär verfolgt werden. Dass es sich bei den verbleibenden Werten 6 bis 13 um Ausdruck einer einzigen konsistenten Werteorientierung handelt, wurde eingangs nicht angenommen.

Anzumerken bleibt, dass die Trennung in eine ökonomische und eine außerökonomische Werteorientierungen ein zwangsläufig unscharfes Konstrukt bleibt, da einzelne Valenzen beide Dimensionen berühren und einer individuell abweichenden Bewertung unterliegen (können). Dabei wird mit der Anführung zweier, eventuell konfligierender Orientierungen nicht eine rationale Erklärung der Organisationswahl verworfen. Zur Modellierung dieser Organisationswahl ist vielmehr ein „weites“ Rational-Choice-Modell besonders gut geeignet, das einen weiter gefassten Blick auf Präferenzen und Restriktionen wirft (vgl. Bamberg et al. 2008: 144; Opp 1999). 


\section{Empirisches Vorgehen und Ergebnisse}

Das empirische Vorgehen zeichnet sich durch ein Bekenntnis zur Methodentriangulation, also einem planvollen, komplementären Wechselspiel von (hier) qualitativen und quantitativen Erhebungsschritten, und einer retrospektiven Ausrichtung aus. Letzteres bedeutet, dass Organisationswählende nach ihrer Entscheidung zu ihren handlungsleitenden Motiven und Werten befragt wurden. Dieses Vorgehen ist mit dem Vorteil verbunden, bereits Sicherheit über das overte Handeln der Befragten zu haben und dadurch leichter Zusammenhänge zwischen Einstellung, Intention und Handlung erfassen zu können.

\subsection{Forschungsdesign}

Das Forschungsdesign besteht in einem ersten Schritt aus qualitativen Interviews, die mit Hilfe eines Leitfragebogens in der Tradition des problemzentrierten Interviews (vgl. Witzel 2000) mit zwanzig Auszubildenden geführt wurden. Die Stichprobenziehung erfolgte angelehnt an das theoretische Sampling (vgl. Glaser/Strauss 1998) und sollte eine Heterogenität bezüglich verschiedener Merkmale der Befragten sicherstellen. Die dadurch produzierten Texte liefern Aufschluss über relevante Valenzen der Organisationswahl und tragen entscheidend zur Sinnrekonstruktion der quantitativ gewonnenen Ergebnisse bei.

Die an das qualitative Design anschließende quantitative Fragebogenerhebung erfasst insgesamt 7.506 Auszubildende an 18 teilnehmenden Berufsschulen der West- und Vorderpfalz (Rheinland-Pfalz). Mit 4.527 ausgewerteten Fragebögen beträgt die Nettorücklaufquote 52,9 Prozent.

\subsection{Ergebnisse der explorativen Faktorenanalyse}

In einem ersten Schritt wird durch das Verfahren der explorativen Faktorenanalyse geprüft, ob sich aus der untereinander korrelierenden Zustimmung zu bestimmten Werten verschiedene Werteorientierungen gewinnen lassen. Zudem soll dadurch empirisch validiert werden, ob den am Ende von Kapitel 2.1 theoriegeleitet gewonnenen Werten tatsächlich eine hinreichend große Nähe attestiert werden kann, um von einer verbindenden ökonomischen Werteorientierung sprechen zu können.

Die Faktorenanalyse als multivariates statistisches Verfahren dient der Dimensionsreduktion. Aus den vorliegenden Valenzen soll damit auf Muster innerhalb dieser Variablen (latente Variablen) geschlossen werden. Die dadurch aufgezeigten Faktoren entsprechen im Rahmen dieser Untersuchung den verschiedenen Werteorientierungen. Der durch diese Faktoren aufgeklärte Varianzanteil beschreibt die Stärke dieser Orientierungen. Die Ergebnisse finden sich in Tabelle 1. Aufgrund mangelnder statistischer Eindeutigkeit, wurde die Valenz Arbeitsmarktmobilität aus der Darstellung entfernt. 


\begin{tabular}{|c|c|c|c|c|c|c|}
\hline \multirow{2}{*}{\multicolumn{2}{|c|}{ Valenz }} & \multicolumn{4}{|c|}{ Faktorladungen } & \multirow{2}{*}{ Kom. } \\
\hline & & 1 & 2 & 3 & 4 & \\
\hline \multicolumn{2}{|l|}{ Übernahmemöglichkeiten } &, 732 & & & & ,583 \\
\hline \multicolumn{2}{|c|}{ Weiterbildungsmöglichkeiten } & ,736 & & & & ,610 \\
\hline \multicolumn{2}{|l|}{$\begin{array}{l}\text { Karriere- und } \\
\text { Entwicklungspotential }\end{array}$} & 667 & & & &, 558 \\
\hline \multicolumn{2}{|c|}{$\begin{array}{l}\text { Vereinbarkeit von Beruf und } \\
\text { Familie }\end{array}$} & ,387 & & & & 399 \\
\hline \multicolumn{2}{|l|}{ Ausbildungsvergütung } & & 670 & & & 553 \\
\hline \multicolumn{2}{|c|}{ Ausbildung mit Gleichaltrigen } & & ,403 & ,467 & & ,431 \\
\hline \multicolumn{2}{|l|}{ Reputation und Image } & &, 544 & & & ,442 \\
\hline \multicolumn{2}{|l|}{ Arbeitsbelastung } & &, 738 & & &, 575 \\
\hline \multicolumn{2}{|c|}{ Internationalität der Ausbildung } & & & ,683 & &, 530 \\
\hline \multicolumn{2}{|c|}{ Vielseitigkeit der Ausbildung } & & & ,631 & & ,478 \\
\hline \multicolumn{2}{|c|}{ Selbstverwirklichungsmöglichkeiten } & & & & ,384 & ,308 \\
\hline \multicolumn{2}{|l|}{ Arbeits- und Betriebsklima } & & & & ,781 & ,624 \\
\hline \multicolumn{2}{|c|}{ Persönlichkeit der Betreuung } & & & & ,605 & ,442 \\
\hline \multirow{2}{*}{\multicolumn{2}{|c|}{$\begin{array}{l}\text { Eigenwerte der rotierten Faktoren } \\
\text { durch Faktor erklärter Varianzanteil } \\
(\%)\end{array}$}} & 2,0 & 1,7 & 1,6 & 1,6 & \\
\hline & & 14,3 & 11,9 & 11,7 & 11,3 & $\sum 49,2$ \\
\hline \multicolumn{7}{|c|}{ Benennung der Werteorientierung (Nummerierung Faktorladungen) } \\
\hline $\begin{array}{c}\text { ökonomische } \\
\text { Werteorientierung }\end{array}$ & \multicolumn{6}{|c|}{$\begin{array}{l}\text { außerökonomische } \\
\text { Werteorientierung }\end{array}$} \\
\hline 1 & \multicolumn{2}{|c|}{2} & \multicolumn{2}{|c|}{3} & \multicolumn{2}{|c|}{4} \\
\hline $\begin{array}{c}\text { Karriere- und } \\
\text { Zukunftsorientierung }\end{array}$ & Freizeito & entierung & \multicolumn{2}{|c|}{$\begin{array}{l}\text { Ausbildungs- } \\
\text { (inhalte)- } \\
\text { orientierung }\end{array}$} & \multicolumn{2}{|c|}{$\begin{array}{l}\text { Wohlfühl- } \\
\text { orientierung }\end{array}$} \\
\hline
\end{tabular}

Tabelle 1: Werteorientierungen der befragten Auszubildenden (eigene Darstellung) ${ }^{6}$

Es liegt das Verfahren der Hauptkomponentenanalyse in der Rotationsmethode Varimax mit Kaiser-Normalisierung zugrunde. Ladungen kleiner als ,380 wurden unterdrückt. Das KaiserMeyer-Olkin-Kriterium erreicht mit ,754 einen auf eine ausreichende Eignung hinweisenden Wert, der Bartlett-Test auf Sphärizität fällt ebenfalls signifikant $(\mathrm{p}<, 001)$, also positiv aus (vgl. Backhaus et al. 2006: 273ff.). Die grafische Darstellung orientiert sich an Diehl/Staufenbiel (2007: 526). Die grundsätzlich ordinal skalierten Daten wurden hier als quasimetrisch aufgefasst. 
Zusätzlich zu den in Kapitel 2.2 aufgeführten Valenzen wird hierbei noch Reputation und Image als eigenständige Valenz abgefragt. Dieser Wert wird an späterer Stelle (Kapitel 3.3) jedoch als eigenständige Modellkomponente (subjektive Norm) weitergeführt.

Erwartungsgemäß zeichnet sich ein Muster ab, das die Valenzen Übernahmemöglichkeiten, Weiterbildungsmöglichkeiten, Karriere- und Entwicklungsmöglichkeiten sowie Vereinbarkeit von Beruf und Familie zu einer latenten Variable, einer Werteorientierung, zusammenfasst. Diese zuvor als ökonomische Werte klassifizierten Orientierungsmomente werden zusammen als Karriere- und Zukunftsorientierung benannt und bezeichnen die am Ausbildungsmarkt systemkonforme, d.h. auf eine Maximierung des Lebenseinkommens abzielende Werteorientierung. Dies jedoch mit der Ausnahme der Valenz Ausbildungsvergütung, die theoriegeleitet ebenfalls zu den ökonomischen Werten gezählt wurde, von den Befragten aber von eben diesen separiert wird. Aufschluss über diesen erklärungsbedürftigen Befund liefern die qualitativen Befragungen, die frühzeitig eine nur sehr geringe Bedeutung dieses Merkmals aufgezeigt haben. Über die sinnrekonstruierend-qualitative Annäherung kann hier zu dem Schluss gelangt werden, dass die Phase der Ausbildung bei der Organisationswahl als Investitionsphase erkannt und konsumtive Erträge in Form des Ausbildungsentgeltes weniger stark gewichtet werden. Von der Höhe des Ausbildungsentgeltes wird nicht auf die Höhe des späteren Einkommens geschlossen.

Als am zweitstärksten vertretene Werteorientierung folgt die in Anlehnung an Rosenstiels sogenannte Freizeitorientierung. Hierunter fallen solche Einzelwerte, die auf einen unmittelbar in der Ausbildung schon zum Tragen kommende positive Effekte auf die außerberufliche (und außermarktliche) Lebenswelt schließen lassen. Wichtig ist hier eine geringe Arbeitsbelastung, die - so zeigen die qualitativen Interviews auf - möglichst wenig mit Wochenendarbeit oder Überstunden einhergeht. Die hohe Bedeutung der Ausbildung zusammen mit Gleichaltrigen ${ }^{7}$ kann über Aussagen plausibilisiert werden, die darauf abzielen, die in der Organisation geknüpften Kontakte auch in der Berufsschule oder außerhalb der Ausbildung pflegen und nutzen zu können. Die hier nun einfließende Relevanz des Ausbildungsentgeltes ist Ausdruck einer materiellen Wertehaltung. Über das Arbeitseinkommen wird hier weniger eine Wertschätzung der eigenen Arbeitsleistung als vielmehr Ressourcen zur Gestaltung der Freizeit symbolisiert. In diesem Sinne ist auch der Wunsch nach einer reputationsstarken Ausbildungsorganisation zu deuten. In den qualitativen Interviews wurde mehrfach angedeutet, dass eine Ausbildung in einer angesehenen Organisation auch das eigene Ansehen im Freundes- und Familienkreis begünstigt.

Vor der Faktorenanalyse wurde geprüft, ob den gesamten Valenzen eine einzelne latente Variable zugrunde liegt. Träfe diese - in anderen Erhebungen erwünschte - Annahme zu, wären verschiedene Werteorientierungen nicht statistisch zu belegen. Die Maßzahl Cronbachs Alpha bestätigt durch einen Wert von, 648 das Vorliegen verschiedener latenter Variablen und damit Werteorientierungen (vgl. Bortz/Döring 2006).

7 Der Wert Ausbildung mit Gleichaltrigen erscheint aufgrund seiner hohen Faktorladung in zwei Orientierungen. 
Neben diesen beiden Werteorientierungen, die bereits durch Rosenstiel aufgezeigt und auch erwartet wurden, kristallisieren sich zwei weitere Orientierungsmuster heraus. Die als solche bezeichnete Ausbildungs(inhalte)orientierung verdeutlicht eine Wertehaltung, die sehr stark auf Arbeitsinhalte ausgerichtet ist. Wichtiger als die Maximierung des Lebenseinkommens erscheint hier die Freude an der Tätigkeit als solcher.

Ähnlich, wenngleich nicht identisch, gestaltet sich die Woblfüblorientierung als vierter Wertehorizont. Hier werden soziale und atmosphärische Vorstellungen geäußert, die auf ein harmonisches, spannungsfreies und persönliches Arbeits- und Ausbildungsverhältnis verweisen. Hier soll im Rahmen der Ausbildung eine Selbstentfaltung stattfinden, die von Vertretern einer Freizeitorientierung mitunter konsequent in die Zeit außerhalb der Ausbildung verlagert wird.

Die aufgefundenen Werteorientierungen korrelieren dabei durchaus untereinander in unterschiedlicher Stärke und verdeutlichen damit eine wertepluralistische Orientierung im Kontext der Berufsausbildung. Während hierbei die Nähe zwischen der Karriereund Zukunftsorientierung auf der einen und der Freizeitorientierung auf der anderen Seite erwartungsgemäß am geringsten bleibt, weisen Freizeitorientierung und Woblfüblorientierung die größte Nähe zueinander auf.

\subsection{Von der Rationalität der Organisationswahl: Einfluss von Einstellung und Werteorientierung auf das Verhalten}

Die Zurückführung des overten Handelns auf eine sich aus einer Werteorientierung herausbildenden Intention gegenüber einer bestimmten Handlung ist nicht voraussetzungslos und kann an dieser Stelle nur in Ansätzen skizziert werden. ${ }^{8}$

Die Modellierung zur empirischen Überprüfung von Konsistenz oder Diskrepanz zwischen empirisch beobachtbarem Verhalten und der von den Befragten kommunizierten Werteorientierung erfolgt über einen werterwartungstheoretischen Ansatz, dessen Anwendung in der auf dem Boden der Rational-Choice-Theorie stehenden mikrofundierten Soziologie diskutiert und für die Empirie herangezogen wird (vgl. Esser 2004: 109ff.; Esser 2002: 227ff.). Vertiefend wird hierbei auf das etablierte sozialpsychologische Verfahren nach Ajzen und Fishbein (1975; 1980; insbesondere auch Ajzen 1991) Bezug genommen, das der werteorientiert bedingten Einstellung noch die subjektive Norm und die wahrgenommene Verhaltenskontrolle gegenüberstellt (vgl. Abbildung 1).

$8 \quad$ Zur Vertiefung siehe Schank (2010), insbesondere die Kapitel 4 sowie 14 bis 16. 


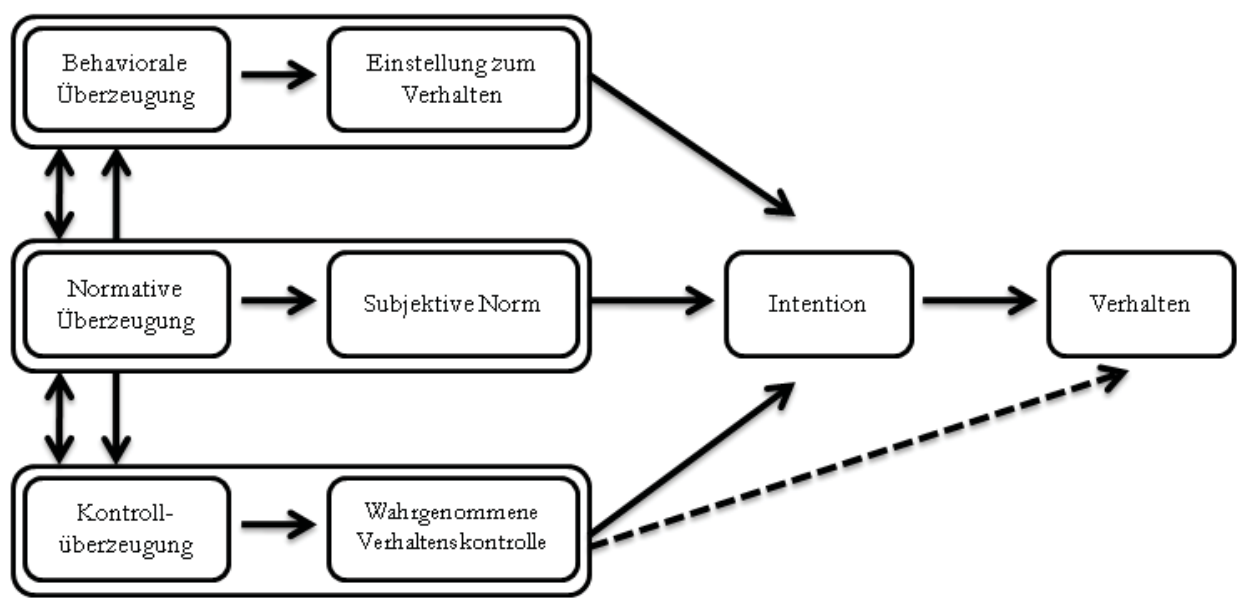

Abbildung 1: Theorie des geplanten Verbaltens (Quelle: in Anlebnung an Ajzen 1991)

Die Einstellung wurde durch die Präferenz bestimmter Valenzen, den Werten, sowie der Instrumentalität verschiedener Organisationstypen gebildet. Eine hohe Instrumentalität bedeutet hierbei, dass die gegebene Organisation in den Augen des Befragten eine hohe Wahrscheinlichkeit aufweist, den jeweiligen Wert in hohem Maße zu erfüllen. Aus der Multiplikation von Valenz und Instrumentalität ergibt sich folglich eine Maßzahl, die im Rahmen eines statistischen Modells eine Prognose des Verhaltens ermöglicht und in dieser retrospektiven Befragung unmittelbar mit dem overten Handeln in Bezug gesetzt werden kann. Die potentiellen Ausbildungsbetriebe als potentielle Organisationen wurden hierbei nach ihrer Unternehmensgröße untergliedert, um die Anzahl der Verhaltensalternativen auf eine handhabbare Größe zu reduzieren. Die zu erklärende Handlung bzw. das Verhalten ist die Bewerbung bei einer Organisation. Der Eintritt in diese Organisation und damit in die duale Berufsausbildung wird hingegen deshalb nicht gewählt, da die anschließenden Rekrutierungsprozesse maßgeblich durch die Organisation fremdbestimmt werden und nicht mehr der Oberhoheit des Akteurs unterliegen.

Die subjektive Norm verdeutlicht die Antizipation von Handlungssanktionen durch relevante Bezugsgruppen oder staatliche Institutionen. Mit ihrer Herauslösung aus den einstellungsbildenden Werten (die es später noch kritisch zu diskutieren gilt) wird der Akteur aus seiner modellimplizierten Isolation gerissen und in einen sozialen Kontext eingebettet. Die Operationalisierung der subjektiven Norm erfolgt an dieser Stelle über die Abfrage von Beliebtheit und Ansehen verschiedener Organisationstypen innerhalb des Freundes- und Familienkreises.

Eine von Ajzen eingeführte, im Rahmen der hier vorliegenden Modellierung aber vernachlässigte, Modellkomponente versucht, die empirisch häufig zu ermittelnde Diskrepanz zwischen Intention und Verhalten aufzuklären. Nicht jede intendierte Handlung kann schlussendlich ausgeführt werden, wie etwa der vielfach verbreitete Wunsch zur Aufgabe des Rauchens bei gleichzeitigem Unvermögen zum Ausführen 
dieser Handlung anschaulich demonstriert. Die hier zu erklärende Handlung, nämlich das Einreichen einer Bewerbung, obliegt aber vollständig der Kontrolle des Akteurs.

Tabelle 2 verdeutlich die regressionsanalytisch gewonnenen Ergebnisse des Grundmodells. Die (lineare) Regressionsanalyse ist ein Analyseverfahren zur Feststellung von Kausalzusammenhängen. Die zu erklärende abhängige Variable, die Bewerbung bei einem bestimmten Organisationstypus, wird versucht auf eine oder mehrere unabhängige Variablen (Werteorientierung und subjektive Norm) zurückzuführen.

\begin{tabular}{|c|c|c|c|c|c|}
\hline \multicolumn{6}{|c|}{ Regressionsmodell zur Erklärung des Verhaltens (Organisationswahl) } \\
\hline \multirow[t]{2}{*}{$\mathrm{n}=3167$} & \multicolumn{5}{|c|}{ multiple lineare Regression } \\
\hline & $\begin{array}{c}\text { B (nicht standardi- } \\
\text { siert) }\end{array}$ & $B$ (standardisiert) & $\mathbf{P}$ & partiell & Teil \\
\hline $\begin{array}{l}\text { Einstellung } \\
\text { (Werteorientierung) }\end{array}$ & 2,032 & ,359 & $<, 001$ & ,344 & ,333 \\
\hline $\begin{array}{l}\text { subjektive Norm } \\
\text { (Image und Reputation) }\end{array}$ &, 505 & ,122 & $<, 001$ & ,124 &, 113 \\
\hline Modellfit ${ }^{9}$ & korrigiertes $R^{2}=, 176$ & & $p<, 001$ & & \\
\hline
\end{tabular}

Tabelle 2: Regressionsmodell zur Erkelärung des Verhaltens (eigene Darstellung)

Beide unabhängigen Variablen sind in ihrem Einfluss ( $($ (standardisiert)) signifikant $(p$ $<, 001)$ und wirken damit nachweislich auf das Verhalten ein. Die Stärke des Einflusses variiert dabei zwischen 0 (kein Beitrag zur Erklärung) und 1 (vollständiger Durchschlag der unabhängigen auf die abhängige Variable). Der Werteorientierung kommt in diesem Modell ein mäßiger, aber spürbarer Einfluss (,359) zu, wohingegen die subjektive Norm ebenfalls ihre Gültigkeit beweist, aber nur schwach zum Tragen kommt $(, 122)$.

Das hier vorgestellte Grundmodell ist in seiner Komplexität überschaubar und berücksichtigt nicht weitere Erklärungsleistungen, die im Rahmen der Untersuchung erbracht wurden. Weitere Untersuchungsschritte integrieren explizit theoriereiche Brückenhypothesen in die Modellierung, die vordergründig auf komplexitätsreduzierende Muster zur Strukturierung der potentiell unbegrenzten Handlungsalternativen abzielen. ${ }^{10}$ Diese weiteren Schritte exponieren die Werteorientierung weiter in ihrer Bedeutung (standardisierte $B$-Werte von bis zu ,498 verweisen auf einen deutlichen Einfluss).

$9 \quad$ Zur Erfassung weiterer Gütekriterien erfolgten Kollinearitätsdiagnose, Residuen-Analyse und Prüfung der Homoskedastizitätsannahme. Die Analyseschritte finden sich bei Schendera (2008: 132ff.).

Besondere Aufmerksamkeit muss dem korrigierten $\mathrm{R}^{2}$ zukommen, das die Streuungserklärung bemisst. Je näher dieser Wert an 1 liegt, desto besser erklärt dieses Modell das Verhalten vollständig. Der hier aufgefundene Wert ist mäßig (,176), aber inhaltlich erklärbar. In diesem Modell werden maßgebliche Einflussfaktoren nicht berücksichtigt. Durch verfeinerte Analyseschritte kann der Wert auf bis zu ,251 erhöht werden. 
Die im hier beschriebenen Modell als eigeneständige Einflussdeterminante aufgefasste subjektive Norm bleibt über alle Regressionsmodelle hinweg von nur geringer oder sogar abzulehnender Relevanz für die Verhaltenserklärung. Dieser Befund spiegelt sich in verschiedenen ähnlich gelagerten Untersuchungen wider und ist Ausdruck einer vielschichtigen Problematik. Während Armitage und Conner (2001) hier noch über theoriereichere und sensiblere Messverfahren diskutieren, erachten ökonomisch respektive mikrofundierte Vertreter wie Esser (2001: 248) die Isolierung der subjektiven Norm als unnötige Verkomplizierung. Jene Normerwartungen werden von ihnen, gleichsam den übrigen Valenzen, in die Nutzenfunktion integriert. Kritisch äußert sich dagegen Stroebe (2008: 117), der sich dem Phänomen der Normbefolgung über eine kognitive Theorie nähert und sie zu einer im Gedächtnis abgespeicherten Repräsentanz erklärt, die durch situative Hinweisreize aktiviert wird und als unkontrollierbare Verhaltensdeterminante wirkt. Der bewussten, rationalen Entscheidung entzieht sie sich damit. Auch Lindenberg (2008: 65) verweist darauf, dass Einstellungen und subjektive Normen keine gleichberechtigten Konstrukte sein können, sondern sich auf unterschiedlichen Bewusstseinsebenen abspielen. Vor diesem Hintergrund wird plausibel, weshalb die subjektive Norm auch in diesem Modell nur einen äußerst geringen Mehrwert zur Verhaltenserklärung liefern kann.

\section{Diskussion}

In einem ersten Schritt rückten die vorhergegangenen Ausführungen die Frage in den Mittelpunkt, an welchen Werten sich Organisationswählende orientieren sollten, um aus einer (bildungs-)ökonomischen Perspektive heraus ein systemkonformes, rationales Verhalten an den Tag zu legen. Die Identifizierung und Prüfung dieser Werte und die damit einhergehende Werteorientierung ist bereits deshalb von einer hohen Relevanz, da gerade ökonomische Studien solche Werte als alleinige Basis für ein rationales Verhalten für zulässig erklären und alternierende Werteorientierungen nur allzu häufig als Ausdruck eines irrationalen oder bedingt rationalen Verhaltens missinterpretieren. Aus der explorativen Faktorenanalyse ging hierbei eine ökonomische Werteorientierung hervor, die einen hohen Deckungsgrad mit den theoretisch gewonnenen Annahmen aufzeigt. Somit kann konstatiert werden, dass der Akteur sich zumindest in seinen Präferenzen durchaus systemkonform verhalten kann, er damit die „Spielregeln“ des Ausbildungs- und Arbeitsmarktes antizipiert und ihnen Folge leistet.

Diese Werteorientierung ist jedoch keineswegs konkurrenzlos, wie jene Orientierungen aufzeigen, die sich überwiegend auf außerökonomische Werte beziehen. Dass diese zumindest nicht unmittelbar monetär bewertbaren Präferenzen in der Mehrzahl der ökonomischen Modellierungen ausgeklammert werden, muss daher als klares Modelldefizit herausgearbeitet werden. Insofern ist dieser Beitrag gleichzeitig als Plädoyer dafür zu begreifen, komplexe Sachverhalte nicht im Geiste einer puristischen Eleganz auf wenige, gut zu operationalisierende und über das Erwerbseinkommen abzubildende Variablen zu reduzieren, sondern bewusst ein weites Rational-Choice-Modell anzustreben.

Dieser Anspruch begründet jedoch zugleich die Grenzen des hier vorliegenden forschungsmethodischen Vorgehens. Gerade die Auswahl nicht-ökonomischer Werte ist zwangsläufig unvollständig und kann - auch wenn sie wie hier empirisch-induktiv 
gewonnen wurden - dem komplexen Wertepluralismus kaum gerecht werden. An dieser Stelle müssen folglich zentrale Überlegungen ausgeblendet werden, wie sie etwa die Mobilitätsbereitschaft, die Attraktivität von Branchen oder Regionen oder der Berufsschule als begleitenden zweiten Lernort betreffen. Auch ein weites RationalChoice-Modell bleibt damit zwangsläufig eine Annäherung und muss die Grenzen der eigenen Aussagekraft herausarbeiten. Im Falle dieses Beitrages werden etwa die regionalen Gelegenheitsstrukturen nicht ausreichend ins Kalkül gezogen. Innerhalb einer Ausbildungsregion steht nur eine begrenzte Anzahl an ausbildenden Organisationen zur Verfügung, die eventuell nicht die Werteorientierung der Organisationswähler widerspiegeln, aber aus Mangel an Alternativen beziehungsweise einer geringen persönlichen Mobilität gewählt werden müssen.

Implikationen für die Praxis besitzen die Befunde, da sie mit einer vermeintlich alleinigen Orientierung an Karriere-, Entwicklungs- und Erwerbspfaden brechen. Dies bedeutet gerade für in der Personalrekrutierung und -entwicklung tätige Unternehmenspraktiker insofern ein gewisses Umdenken, da die (potentiellen) Organisationsmitglieder über eine rein ökonomische Anreizstruktur weder nachhaltig zu gewinnen noch zu motivieren sind. Es werden verschiedene Typen von Organisationswählenden aufgezeigt, die mit ihrer Organisationszugehörigkeit unter Umständen Motive verfolgen, die nicht unbedingt im Einklang mit jenen der Organisation stehen müssen. Besonders hervor sticht bei diesen Überlegungen der Typus des Freizeitorientierten, der sein berufliches Engagement primär als leidvoll betrachtet und die persönliche Erfüllung allein außerhalb der Organisation sucht. Hier droht die Gefahr von Fehlbesetzungen, da zwar besonders motivierte und entwicklungsinteressierte Organisationsmitglieder nachgefragt werden, der Freizeitorientierte sein organisationales Engagement aber möglichst gering halten möchte.

Aus einer unternehmensethischen Perspektive sind die Ergebnisse in die Diskussion um die Unternehmenskultur als Ausdruck „tiefenstruktureller Werte“ (Mayrhofer/Meyer 2004: 1026) einzuordnen. So wie jede Organisation neben ihren kodifizierten Werten und Visionen auch informelle, mitunter heimliche Spielregeln kennt, können auch Organisationswählende Werte teilen und Ziele anstreben, die in den bisherigen Auswahlprozessen nicht hinreichend expliziert werden können und daher der Organisation verborgen bleiben. Wird in solchen Prozessen nicht offen mit unterschiedlichen Orientierungen umgegangen, drohen beide Seiten missverständliche Signale auszusenden und ein kulturelles Mismatch einzugehen. Eine Empfehlung kann lauten, sich bewusst um eine Offenlegung kultureller Muster (vgl. Göbel 2010: 225) zu bemühen und somit frühzeitig Transparenz darüber herzustellen, welche Werteorientierung in der Organisation gelebt werden kann.

\section{Literaturverzeichnis}

Ajzen, I. (1991): The Theory of Planned Behavior. Some Unresolved Issues. Organizational Behavior and Human Decision Processes, Vol. 50/No. 2, 179-211.

Ajzen, I./ Fishbein, M. (1975): Belief, Attitude, Intention, Behavior, Reading, Massachusetts: Addison-Wesley.

Ajzen, I./ Fishbein, M. (1980): Understanding Attitudes and Predicting Social Behavior, Englewood Cliff, New Jersey: Prentice-Hall. 
Allehoff, W. (1985): Berufswahl und berufliche Interessen, Göttingen et al: Hogrefe AG.

Armitage, C. J./ Connor, M. (2001): Efficacy of the Theory of Planned Behaviour: A MetaAnalytic Review, in: British Journal of Social Psychology, Vol. 40/No. 4, 471-499.

Austen, S. (1997): Occupational Choice and Choosing to Become an Apprentice, Centre for Labour Market Research/Curtin University of Technology, Discussion Paper.

Backhaus, K./Plinke, W./Erichson, B. (2006): Multivariate Analysemethoden - Eine anwendungsorientierte Einführung, Berlin: Springer.

Bamberg, S./ Davidov, E./ Schmidt, P. (2008): Wie gut erklären „enge“ oder „weite“ RationalChoice-Versionen Verhaltensänderungen? Ergebnisse einer experimentellen Interentionsstudie, in: Diekmann, A./ Eichner, K./ Schmidt, P./ Voss, T. (Hrsg.): Rational Choice: Theoretische Analysen und empirische Resultate, Wiesbaden: VS Verlag für Sozialwissenschaften, 144-169.

Beck, U./ Brater, M./ Daheim, J. (1980): Soziologie der Arbeit und der Berufe: Grundlagen, Problemfelder, Forschungsergebnisse, Reinbek bei Hamburg: Rohwolt.

Beck, U./ Brater, M./ Wegener, B. (1979): Berufswahl und Berufszuweisung. Zur sozialen Verwandtschaft von Ausbildungsberufen, Frankfurt a. M./New York: Campus Verlag.

Becker, G. S. (1993): Ökonomische Erklärung menschlichen Verhaltens, Tübingen: Mohr.

Becker, G. S. (2008): Economic Theory, New Brunswick/New Jersey: Aldine Transaction.

Beinke, L. (2006): Berufswahl und ihre Rahmenbedingungen. Entscheidungen im Netzwerk der Interessen, Frankfurt a. M: Peter Lang.

Berthon, P./ Ewing, M./ Hah, L. (2005): Captivating Company: Dimension of Attractiveness in Employer Branding, in: International Journal of Advertising, Vol. 24/No. 2, 151-172.

Bortz, J./ Döring, N. (2006): Forschungsmethoden und Evaluation für Human- und Sozialwissenschaftler, Berlin: Springer.

Bourdieu, P. (1983): Ökonomisches Kapital, kulturelles Kapital, soziales Kapital, in: Kreckel, R. (Hrsg.): Soziale Ungleichheiten, Soziale Welt, Sonderband 2, Göttingen: Schwartz, 183198.

Bourdieu, P. (1998): Practical Reason, On the Theory of Action, Stanford: University Press.

Diebl, J. M./ Staufenbiel, T. (2007): Statistik mit SPSS für Windows, Version 15, Eschborn: Klotz.

Dubin, R. (1956): Industrial Workers`Worlds: A Study of the "Central Life Interests" of Industrial Workers, in: Social Problems, Vol. 3/No. 3, 131-142.

Dubin, R./ Goldmann, D. R. (1972): Central Life Interests of American Middle Managers and Specialists, in: Journal of Vocational Behavior, Vol. 2/No. 2, 133-141.

Ebbingshaus, M. (2011): Different routes - one goal: how companies recruit apprentices, in: Berufsbildung in Wissenschaft und Praxis, Vol. 40, Special Edition "Trends in German VET 2011/2012", 14-18.

Esser, H. (2000): Soziologie. Spezielle Grundlagen, Bd. 2: Die Konstruktion der Gesellschaft, Frankfurt a. M./New York: Campus.

Esser, H. (2001): Soziologie. Spezielle Grundlagen, Bd. 6: Sinn und Kultur, Frankfurt a. M./New York: Campus.

Esser, H. (2002): Soziologie. Spezielle Grundlagen, Bd. 1: Situationslogik und Handeln, Frankfurt a. M./New York: Campus.

Esser, H. (2004): Soziologische Anstöße, Frankfurt a. M./New York: Campus.

Friedberg, R. (2000): You can't take it with you? Immigrant Assimilation and the Portability of Human Capital, in: Journal of Labor Economics, Vol. 18/No. 2, 221-251.

Frommberger, D. (2007): Berufsausbildung in Deutschland vor dem Hintergrund europäischer Entwicklungen, in: Dietrich, H./ Severing, E. (Hrsg.): Zukunft der dualen Berufsaus- 
bildung - Wettbewerb der Bildungsgänge, Schriften zur Berufsbildungsforschung der Arbeitsgemeinschaft Berufsbildungsforschungsnetz (AG BFN), Bielefeld: Bundesinstitut für Berufsbildung, 143-161.

Gertz, W. (2011): Nachwuchswerbung zwischen Hoffen und Bangen, in: Personalwirtschaft: Magazin für Human Resources, Jg. 2011/Heft 5, 12-14.

Glaser, B./ Strauss, A. L. (1998): Grounded Theory. Strategien qualitativer Forschung, Berlin: Huber.

Göbel, E. (2010): Unternehmensethik, Stuttgart: Lucius \& Lucius.

Hinz, T./ Abraham, M. (2005): Theorien des Arbeitsmarktes: Ein Überblick, in: Abraham, M./ Hinz, T. (Hrsg.): Arbeitsmarktsoziologie. Probleme, Theorien, empirische Befunde, Wiesbaden: VS Verlag für Sozialwissenschaften, 17-69.

Kluckhohn, C. (1951): Values and Value-Orientation in the Theory of Action: An Exploration in Definition and Classification, in: Parsons, T./ Shils, E. (Eds.): Toward a General Theory of Action, Cambridge/Mass: Harvard University Press, 388-433.

Lange, E. (1978): Berufswahl. Eine empirische Untersuchung der Berufswahlsituation von Hauptschülern, Realschülern und Abiturienten, München: Fink.

Lappe, L. (2006): Jugend in der Berufsbildung. Arbeitsmarkt- und Berufsforschung, in: Arnold, R./ Lipsmeier, A. (Hrsg.): Handbuch der Berufsbildung, Wiesbaden: VS Verlag für Sozialwissenschaften, 73-84.

Lieber, B. (1995): Personalimage. Explorative Studien zum Image und zur Attraktivität von Unternehmen als Arbeitgeber, München und Mehring: Hampp.

Lindenberg, S. (2008): Social Norms: What Happens When They Become More Abstract?, in: Diekmann, A./ Eichner, K./ Schmidt, P./ Voss, T. (Eds.): Rational Choice: Theoretische Analysen und empirische Resultate, Wiesbaden: VS Verlag für Sozialwissenschaften, 63-83.

Maslow, A. H. (1943): A Theory of Human Motivation, in: Psychological Review, Vol. 50/No. 4, 370-396.

Mayrhofer, W./ Meyer, M. (2004): Organisationskultur, in: Schreyögg, G./ von Werder, A. (Hrsg.): Handwörterbuch Unternehmensführung und Organisation, Stuttgart: Schäffer/Poeschel, 1025-1033.

Neubäumer, R. (1999): Der Ausbildungsstellenmarkt der Bundesrepublik Deutschland. Eine theoretische und empirische Analyse, Berlin: Duncker \& Humblot.

Nowak, G. (2002): Berufswahl. Theorie und Praxis bei LehrabsolventInnen, Wien: My choice solution.

Opp, K.-D. (1999): Contending Conceptions of The Theory of Rational Choice, in: Journal of Theoretical Politics, Vol. 11/No. 2, 171-202.

Rosenstiel, L. v. (1982): Karriere? Nein danke!, in: IBM-Nachrichten, Jg. 32/Heft 261, 32-61.

Rosenstiel, L. v./ Stengel, M. (1987): Identifikationskrise? Zum Engagement in betrieblichen Führungspositionen, Bern et al.: Huber.

Rosenstiel, L. v./ Nerdinger, F. W. (2000): Die Münchner Wertestudien. Überblick und (vorläufiges) Resume. In: Psychologische Rundschau, Jg. 51/Heft 3, 146-157.

Sager, F. (2008): Securing the Long-Term Bases of the Dual System: A Realistic Evaluation of Apprenticeship Marketing in Switzerland, in: Journal of Vocational Education and Training, Vol. 60/No. 3, 327-341.

Scharmann, T. (1965): Jugend in Arbeit und Beruf, Weinheim/München: Juventa-Verlag.

Schank, C. (2010): Die Betriebswahl im dualen System der Berufsausbildung. Eine empirische Analyse aus mittelstandsökonischer Perspektive, Wiesbaden: VS Verlag für Sozialwissenschaften. 
Schein, E. H. (1980): Organisationspsychologie, Wiesbaden: Gabler.

Schendera, C. F. (2008): Regressionsanalyse mit SPSS, München: Oldenburg.

Schmalen, H. (1973): Rationale individuelle Berufswahl und die langfristige Entwicklung des Arbeitsmarktes, in: Zeitschrift für die gesamte Staatswissenschaft, Jg. 129/Heft 2, 292311.

Sheldon, G. (1986): Bestimmungsgrößen der Berufswahl, in: Schelbert-Syfrig, H./ Blattner, H./ Halbherr, P./ Harabi, N. (Hrsg.): Mikroökonomik des Arbeitsmarktes: Theorie, Methoden und empirische Ergebnisse für die Schweiz, Bern: Paul Haupt Verlag, 327-384.

Simon, H./ Wiltinger, K./ Sebastian, K./ Tacke, G. (1995): Effektives Personalmarketing - Strategien, Instrumente, Fallstudien, Wiesbaden: Capitum.

Stroebe, W. (2008): Wann und wie beeinflussen Normen das Verhalten: Eine sozial-kognitive Analyse, in: Diekmann, A./ Eichner, K./ Schmidt, P./ Voss, T. (Hrsg.): Rational Choice: Theoretische Analysen und empirische Resultate, Wiesbaden: VS Verlag für Sozialwissenschaften, 101-119.

Sutherland, M. M./ Torricelli, D. G./ Karg, R. F. (2002): Employer-of-Choice Branding for Know-ledge Workers, in: South African Journal of Business Management, Vol. 33/Nr. 4, 13-20.

Ulrich, P. (2005): Zivilisierte Marktwirtschaft. Eine wirtschaftsethische Orientierung, Freiburg: Resch.

Witzel, A. (2000): Das problemzentrierte Interview [25 Absätze]. Forum Qualitative Sozialforschung/Forum Qualitative Social Research, 1 (1), Art. 22. Link: http://nbn-resolving. de/urn:nbn:de:0114-fqs0001228 (zuletzt abgerufen am 15.06.2009). 